\title{
ON GENERALIZED THERMOELASTIC DISTURBANCES IN AN ELASTIC SOLID WITH A SPHERICAL CAVITY1
}

\author{
BASUDEB MUKHOPADHYAY \\ Department of Mathematics \\ Bengal Engineering College \\ Howarh - 711103, West Bengal, INDIA \\ RASAJIT BERA \\ Department of Mathematics \\ Presidency College \\ Calcutta - 700 073, INDIA \\ LOKENATH DEBNATH \\ Department of Mathematics \\ University of Central Florida \\ Orlando, Florida 32816, USA
}

\begin{abstract}
In this paper, a generalized dynamical theory of thermoelasticity is employed to study disturbances in an infinite elastic solid containing a spherical cavity which is subjected to step rise in temperature in its inner boundary and an impulsive dynamic pressure on its surface. The problem is solved by the use of the Laplace transform on time. The short time approximations for the stress, displacement and temperature are obtained to examine their discontinuities at the respective wavefronts. It is shown that the instantaneous change in pressure and temperature at the cavity wall gives rise to elastic and thermal disturbances which travel with finite velocities $\nu_{1}$ and $\nu_{2}\left(>\nu_{1}\right)$ respectively. The stress, displacement and temperature are found to experience discontinuities at the respective wavefronts. One of the significant findings of the present analysis is that there is no diffusive nature of the waves as found in classical theory.
\end{abstract}

Key words: Generalized thermoelastic disturbances, thermal and elastic waves, discontinuities of stress, displacement and temperature.

AMS (MOS) subject classifications:73D20, 73D30.

\footnotetext{
${ }^{1}$ Received: October, 1990. Revised: April, 1991.
} 


\section{INTRODUCTION}

Lord and Shulman [1] have first initiated the study of the generalized dynamical theory of thermoelasticity by introducing a relaxation constant to take into account the time needed for acceleration of the heat flow. If the relaxation constant is set equal to zero, Lord and Shulman's equations reduce to the classical field equations for the linear coupled dynamical theory of thermoelasticity (see, for example, Boley and Weiner [2], Chapter 1). Lord and Shulman's analysis results in a system of coupled hyperbolic partial differential equations which is then used for the investigation of a particular problem in order to find a closed form solution.

Subsequently, considerable attention has been given to the study of wave propagation in generalized thermoelasticity based upon the work of Lord and Shulman. Several authors including Norwood and Warren [3], McCarthy [4], Puri [5], Agarwal [6], Chandrasekharaiah et al. [7-8], Roy-Choudhuri and Debnath [9-10] have investigated various problems revealing interesting phenomena which characterize the generalized thermoelasticity.

On the other hand, Nariboli [11] has considered the problem of a spherically symmetric thermal shock in an infinite elastic medium with a spherical cavity. Matsumoto and Nakahara [12] have solved the problem of a spherically symmetric thermal shock in an infinite medium with a spherical cavity. In spite of these studies, these problems have hardly received any attention within the scope of the generalized thermoelasticity.

The main objective of this paper is to make an investigation of a coupled problem of a thermoelastic infinite solid containing a spherical cavity which is subjected to step rise in temperature in its inner boundary and an impulsive or step rise in dynamic pressure on its surface. The short-time approximations for the stress, displacement and temperature are obtained using the Laplace transform technique. It is shown that the instantaneous change in pressure and temperature at the cavity-wall gives rise to elastic and thermal disturbances which travel in the medium with finite velocities $v_{1}$ and $v_{2}\left(>v_{1}\right)$ respectively. The stress, displacement, and temperature are found to experience discontinuities at the respective wavefronts. One of the significant findings of this analysis is that there is no diffusive nature of the waves as found in classical theory.

\section{BASIC EQUATIONS OF GENERALIZED THERMOELASTICITY}

We consider an infinite isotropic homogeneous elastic medium with a spherical cavity of radius $r=a$. We denote the radial and circumferential stresses and strains by 
$\sigma_{r r}, \sigma_{\theta \theta}, \sigma_{\phi \phi}$ and $e_{r r}, e_{\theta \theta}, e_{\phi \phi}$ respectively. Invoking the spherical symmetry, the radial displacement $u$ is the only displacement and the stress and strain tensors are independent of $\theta$ so that we have the following relations

$$
\sigma_{\theta \theta}=\sigma_{\phi \phi}, \quad e_{r r}=\frac{d u}{d r}, \quad e_{\theta \theta}=e_{\psi \phi}=\frac{u}{r}
$$

The stress-strain relations are

$$
\begin{gathered}
\sigma_{r r}=\lambda \Delta+2 \mu e_{r r}-\alpha(3 \lambda+2 \mu) T \\
\sigma_{\theta \theta}=\sigma_{\phi \phi}=\lambda \Delta+2 \mu e_{\theta \theta}-\alpha(3 \lambda+2 \mu) T
\end{gathered}
$$

where $\Delta=e_{r r}+e_{\theta \theta}+e_{\phi \phi}$ is the dilatation, $\lambda, \mu$ are Lame's constants, $\alpha$ is the coefficient of linear expansion, and $T$ is the excess of temperature over the reference temperature $T_{0}^{*}$ when the medium is unstressed.

The generalized heat conduction equation with thermal relaxation due to Lord and Shulman [1] is given by

$$
\kappa \nabla_{1}^{2} T=\rho C_{\mathrm{v}}(\dot{T}+\tau \ddot{T})+\beta T_{0}^{*}(\dot{\Delta}+\tau \ddot{\Delta})
$$

where $\kappa$ is the coefficient of thermal conductivity of the solid, $\rho$ is the constant mass density of the medium, $C_{\mathrm{v}}$ is the specific heat of the solid at constant strain, $\tau$ is the thermal relaxation time, $\beta=(3 \lambda+2 \mu) \alpha$, and $\nabla_{1}^{2} \equiv\left(\frac{\partial}{\partial r}+\frac{2}{r}\right) \frac{\partial}{\partial r}$.

The stress equation of motion is

$$
\frac{\partial}{\partial r} \sigma_{r r}+\frac{2}{r}\left(\sigma_{r r}-\sigma_{\theta \theta}\right)=\rho \frac{\partial^{2} u}{\partial t^{2}}
$$

It is convenient to introduce the following dimensionless quantities

$$
\begin{gathered}
(U, R)=\frac{1}{a}(u, r), \quad \Theta=T / T_{0}^{*}, \quad \eta=\mathrm{v} t / a, \\
\left(\sigma_{R}, \sigma_{\theta}, \sigma_{\phi}\right)=\frac{1}{2 \mu}\left(\sigma_{r r}, \sigma_{\theta \theta}, \sigma_{\phi \phi}\right),
\end{gathered}
$$

where $v^{2}=(\lambda+2 \mu) / \rho$ is the dilatational wave speed in the medium. In terms of the dimensionless quantities, equations (2.2)-(2.5) can be written as

$$
\sigma_{R}=\frac{\partial U}{\partial R}+\frac{\lambda}{2 \mu}\left(\frac{\partial U}{\partial R}+\frac{2 U}{R}\right)-\frac{\beta T_{0}^{*}}{2 \mu} \Theta
$$




$$
\begin{gathered}
\sigma_{\theta}=\sigma_{\phi}=\frac{U}{R}+\frac{\lambda}{2 \mu}\left(\frac{\partial U}{\partial R}+\frac{2 U}{R}\right)-\frac{\beta T_{0}^{*}}{2 \mu} \Theta \\
\frac{\partial^{2} \Theta}{\partial R^{2}}+\frac{2}{R} \frac{\partial \Theta}{\partial R}=a_{2}\left(\frac{\partial \Theta}{\partial \eta}+\frac{\tau \mathrm{v}}{a} \frac{\partial^{2} \Theta}{\partial \eta^{2}}\right)+a_{3}\left(\frac{\partial \Delta}{\partial \eta}+\frac{\tau \mathrm{v}}{a} \frac{\partial^{2} \Delta}{\partial \eta^{2}}\right), \\
\frac{\partial \sigma_{R}}{\partial R}+\frac{2}{R}\left(\sigma_{R}-\sigma_{\theta}\right)=\frac{\rho v^{2}}{2 \mu} \frac{\partial^{2} U}{\partial \eta^{2}}
\end{gathered}
$$

where $a_{2}=\left(\frac{\rho C_{v}}{\kappa}\right) a v$ and $a_{3}=\beta a v / \kappa$ are dimensionless constants. Making use of equations (2.8)-(2.9) and (2.11), we finally obtain the following equations

$$
\begin{gathered}
D D_{1} U-a_{1} D \Theta=\frac{\partial^{2} U}{\partial \eta^{2}} \\
D_{1} D \Theta-a_{2}\left(\frac{\partial \Theta}{\partial \eta}+\tau^{\prime} \frac{\partial^{2} \Theta}{\partial \eta^{2}}\right)=a_{3}\left(\frac{\partial}{\partial \eta}+\tau^{\prime} \frac{\partial^{2}}{\partial \eta^{2}}\right)\left(D_{1} U\right),
\end{gathered}
$$

where $a_{1}=\left(\beta T_{0}^{*}\right) /(\lambda+2 \mu)$ is another dimensionless constant, $\tau^{\prime}=\tau \mathrm{v} / a$, and

$$
D \equiv \frac{\partial}{\partial R} \text { and } D_{1} \equiv \frac{\partial}{\partial R}+\frac{2}{R}
$$

We assume the region $S_{0}: r \geq a$ is initially at rest and to have zero temperature and zero velocity. It is noted that this condition is not required in the classical theory, but it is an essential requirement for the solution of the present problem.

\section{THE SOLUTION OF THE PROBLEM AND BOUNDARY CONDITIONS}

It is convenient to solve the problem by introducing the Laplace transforms (Myint-U and Debnath [13]) $\bar{U}(R, s)$ and $\bar{\Theta}(R, s)$ by

$$
[\bar{U}, \bar{\Theta}]=\int_{0}^{\infty} e^{-s \eta}[U, \Theta] d \eta, \quad \operatorname{Re} s>0
$$

Application of the transform method with the prescribed initial conditions $U(R, 0)=\Theta(R, 0)=0$, equations (2.13)-(2.14) become

$$
\begin{aligned}
\left(D D_{1}-s^{2}\right) \bar{U} & =a_{1} D \bar{\Theta} \\
\left(D_{1} D-a_{2} s-\tilde{\beta} s^{2}\right) \bar{\Theta} & =a_{3}\left(s+\tau^{\prime} s^{2}\right) D_{1} \bar{U}
\end{aligned}
$$


where $\tilde{\beta}=a_{2} \tau^{\prime}=\tau /\left(\frac{k}{v^{2}}\right)$ is the dimensionless relaxation time (or relaxation constant) and $k=\left(\kappa / \rho C_{\mathrm{v}}\right)$ is the thermal diffusivity.

Invoking operations by $D D_{1}$ and $D_{1} D$ respectively to (3.2)-(3.3) and using the remaining one, it gives the following equations

$$
\begin{aligned}
\left(L-m_{1}^{2}\right)\left(L-m_{2}^{2}\right) \bar{U}=0 \\
\left(M-m_{1}^{2}\right)\left(M-m_{2}^{2}\right) \bar{\Theta}=0
\end{aligned}
$$

where $L \equiv D D_{1}$ and $M \equiv D_{1} D$ are two operators, and $m_{1}^{2}, m_{2}^{2}$ are the roots of the quadratic in $m^{2}$ given by

$$
m^{4}-\left\{s^{2}(1+\tilde{\beta}+\varepsilon \tilde{\beta})+s a_{2}(1+\varepsilon)\right\} m^{2}+\left(a_{2} s^{3}+\tilde{\beta} s^{4}\right)=0
$$

where

$$
\varepsilon=\frac{a_{1} a_{3}}{a_{2}}=\left(\beta^{2} T_{0}^{*}\right) /\left(\rho C_{v}\right)(\lambda+2 \mu)
$$

is the thermoelastic coupling constant.

The solutions of equation (3.4) are modified spherical Bessel functions of order $3 / 2$, and can be expressed in terms of exponentials. Thus we have

$$
\bar{U}=\sum_{i=1}^{2} A_{i} e^{-m_{i} R}\left(\frac{1}{R}+\frac{1}{m_{i} R^{2}}\right) .
$$

Similarly, the solution of equation (3.5) can be obtained as

$$
\bar{\odot}=\sum_{i=1}^{2} B_{i} \cdot \frac{e^{-m_{i} R}}{R},
$$

where $A_{i}$ and $B_{i}$ are functions of $s$. Since the solutions must vanish at infinity, the square roots are always so chosen that they have positive real parts.

The boundary conditions of the problem are:

(i) The boundary of the spherical cavity of radius $a$ is subjected to a dynamical pressure so that $\sigma_{R}=-P(\eta)$ on $R=1$, for $\eta \geq 0$, and

(ii) A step rise in temperature of magnitude $\chi$ is applied at the inner boundary of the cavity so that $\quad \odot=\chi \cdot H(\eta)$ on $R=1$, for $\eta \geq 0$,

where $H(\eta)$ is the Heaviside unit function, $\chi$ being constant.

These will give for $R=1$, 


$$
\bar{\odot}=\frac{\chi}{s} \text { and } \bar{\sigma}_{R}=-\bar{P}(s)
$$

Introducing (3.8)-(3.9) into equation (3.2) (for $R=1$ ), we have

$$
B_{i}=\frac{s^{2}-m_{i}^{2}}{a_{1} m_{i}} \cdot A_{i}
$$

From (3.8)-(3.10), we have

$$
A_{1} \frac{e^{-m_{1}}}{m_{1}} \cdot\left(s^{2} a_{4}+2 m_{1}+2\right)+A_{2} \cdot \frac{e^{-m_{2}}}{m_{2}}\left(s^{2} a_{4}+2 m_{2}+2\right)=\bar{P}(s),
$$

and

$$
A_{1} \cdot \frac{s^{2}-m_{1}^{2}}{a_{1} m_{1}} \cdot e^{-m_{1}}+A_{2} \cdot \frac{s^{2}-m_{2}^{2}}{a_{1} m_{2}} \cdot e^{-m_{2}}=\chi / s
$$

Solving (3.12) and (3.13), we obtain

$$
\begin{aligned}
\bar{\sigma}_{\theta}=\bar{\sigma}_{\phi}=- & \frac{e^{-m_{1} R_{1}}}{\Delta^{\prime} R^{3}}\left[\bar{P}(s)\left(s^{2}-m_{2}^{2}\right)-\frac{\chi a_{1}}{s} \cdot\left(s^{2} a_{4}+2 m_{2}+2\right)\right] \cdot\left[a_{4} s^{2} R^{2}-m_{1}^{2} R^{2}-m_{1} R-1\right] \\
& +\frac{e^{-m_{2} R_{1}}}{\Delta^{\prime} R^{3}} \cdot\left[\bar{P}(s)\left(s^{2}-m_{1}^{2}\right)-\frac{\chi a_{1}}{s}\left(s^{2} a_{4}+2 m_{1}+2\right)\right]\left[a_{4} s^{2} R^{2}-m_{2}^{2} R^{2}-m_{2} R-1\right],
\end{aligned}
$$

and

$$
\begin{aligned}
\bar{\sigma}_{R}= & -\frac{e^{-m_{1} R_{1}}}{\Delta^{\prime} R^{3}} \cdot\left[\bar{P}(s)\left(s^{2}-m_{2}^{2}\right)-\frac{\chi a_{1}}{s} \cdot\left(s^{2} a_{4}+2 m_{2}+2\right)\right] \cdot\left[a_{4} s^{2} R^{2}+2 m_{1} R+2\right] \\
& +\frac{e^{-m_{2} R_{1}}}{\Delta^{\prime} R^{3}} \cdot\left[\bar{P}(s)\left(s^{2}-m_{1}^{2}\right)-\frac{\chi a_{1}}{s} \cdot\left(s^{2} a_{4}+2 m_{1}+2\right)\right] \cdot\left[a_{4} s^{2} R^{2}+2 m_{2} R+2\right]
\end{aligned}
$$

where $R_{1}=R-1, a_{4}=1+(\lambda / 2 \mu)$ and $\Delta^{\prime}=\left(m_{1}-m_{2}\right)\left[\left(a_{4} s^{2}+2\right)\left(m_{1}+m_{2}\right)+2\left(s^{2}+m_{1} m_{2}\right)\right]$.

The exact evaluation of the Laplace inverse transform is almost a formidable task. Therefore, special attention will be given to the possible discontinuities and short time approximations. We express the roots of (3.6) in the form

$$
m_{1,2}^{2}=\left(\frac{\sqrt{\alpha} \pm \sqrt{\gamma}}{2}\right)^{2}
$$

where 


$$
\alpha, \gamma=s^{2}(1+\tilde{\beta}+\varepsilon \tilde{\beta})+s a_{2}(1+\varepsilon) \pm 2\left(a_{2} s^{3}+\tilde{\beta} s^{4}\right)^{\frac{1}{2}}
$$

Putting $s=\frac{1}{p}$, we can write

$$
m_{1,2}=\frac{\psi_{1}(p) \pm \psi_{2}(p)}{2 p}
$$

where

$$
\psi_{1,2}(p)=\left[(1+\tilde{\beta}+\varepsilon \tilde{\beta})+p a_{2}(1+\varepsilon) \pm 2\left(\tilde{\beta}+a_{2} p\right)^{\frac{1}{2}}\right]^{\frac{1}{2}}
$$

Expanding by means of Maclaurin's series and taking $p=\frac{1}{s}$, we have

$$
m_{1,2}=\mu_{1,2}+\frac{s}{v_{1,2}}+\frac{\eta_{1,2}^{\prime}}{s}+0\left(\frac{1}{s^{2}}\right)
$$

where

$$
\begin{aligned}
\mu_{1,2} & =\frac{a_{2}}{4} \cdot\left[\frac{(1+\varepsilon)+1 / \sqrt{\tilde{\beta}}}{\{1+(1+\varepsilon) \tilde{\beta}+2 \sqrt{\tilde{\beta}}\}^{\frac{1}{2}}} \pm \frac{(1+\varepsilon)-1 / \sqrt{\tilde{\beta}}}{\{1+(1+\varepsilon) \tilde{\beta}-2 \sqrt{\tilde{\beta}}\}^{\frac{1}{2}}}\right], \\
\frac{1}{v_{1,2}}= & \frac{1}{2}\left[\{1+(1+\varepsilon) \tilde{\beta}+2 \sqrt{\tilde{\beta}}\}^{\frac{1}{2}} \pm\{1+(1+\varepsilon) \tilde{\beta}-2 \sqrt{\tilde{\beta}}\}^{\frac{1}{2}}\right], \\
\eta_{1,2}^{1}= & -\frac{a_{2}^{2}}{16 \tilde{\beta}^{\frac{3}{2}}} \cdot\left[\{1+(1+\varepsilon) \tilde{\beta}+2 \sqrt{\tilde{\beta}}\}^{-\frac{1}{2}} \mp\{1+(1+\varepsilon) \tilde{\beta}-2 \sqrt{\tilde{\beta}}\}^{-\frac{1}{2}}\right] \\
& -\frac{a_{2}^{2}}{16} \cdot\left[\frac{\left\{(1+\varepsilon)+\tilde{\beta}^{-\frac{1}{2}}\right\}^{2}}{\left\{\{1+(1+\varepsilon) \tilde{\beta}+2 \sqrt{\tilde{\beta}}\}^{\frac{3}{2}} \pm \frac{\left\{(1+\varepsilon)-\tilde{\beta}-\frac{1}{2}\right\}^{2}}{\{1+(1+\varepsilon) \tilde{\beta}-2 \sqrt{\tilde{\beta}}\}^{\frac{3}{2}}}\right] .}\right.
\end{aligned}
$$

Since the relaxation time is small, an attention will be given to small time approximations. We next use Abel's theorem (see Doetsch [14]) stating that $\lim _{\eta \rightarrow 0} f(\eta)=\lim _{s \rightarrow \infty} s \bar{f}(s)$ 
in order to calculate approximate values of stresses.

In order to illustrate the general theory, we now consider two special cases:

I. $P(\eta)=P_{0} \delta(\eta)$, II. $P(\eta)=P_{0}^{\prime} H(\eta)$ where $P_{0}$ and $P^{\prime}{ }_{0}$ are constants and $\delta(x)$ is the Dirac function of distribution.

CASE I. We expand the expressions for $\bar{\sigma}_{\theta}$ and $\bar{\sigma}_{R}$ in ascending powers of $\frac{1}{s}$ and retain all terms up to $\frac{1}{s^{2}}$. So, for large $s$, we obtain

$$
\begin{gathered}
\bar{\sigma}_{\theta}=\bar{\sigma}_{\phi} \sim-\frac{e^{-R_{1}\left(\frac{s}{v_{1}}+\mu_{1}\right)}}{R^{3} a_{4}\left(\frac{1}{v_{1}^{2}}-\frac{1}{v_{2}^{2}}\right)} \cdot\left[L_{1}+\left(L_{1} L_{4}+L_{2}\right) \frac{1}{s}+\left(L_{3}+L_{1} L_{5}+L_{2} L_{4}\right) \frac{1}{s^{2}}\right] \\
+\frac{e^{-R_{1}\left(\frac{s_{2}}{v_{2}}+\mu_{2}\right)}}{R^{3} \cdot a_{4}\left(\frac{1}{v_{1}^{2}}-\frac{1}{v_{2}^{2}}\right)} \cdot\left[L^{\prime}{ }_{1}+\left(L_{1}^{\prime} L_{4}+L_{2}^{\prime}\right) \frac{1}{s}+\left(L^{\prime}{ }_{3}+L_{1}^{\prime} L_{5}+L_{2}^{\prime} L_{4}\right) \frac{1}{s^{2}}\right],
\end{gathered}
$$

and

$$
\begin{aligned}
\bar{\sigma}_{R} \sim-\frac{e^{-R_{1}\left(\frac{s}{v_{1}}+\mu_{1}\right)}}{R^{3} a_{4}\left(\frac{1}{v_{1}^{2}}-\frac{1}{v_{2}^{2}}\right)} \cdot\left[L_{6}+\left(L_{6} L_{4}+L_{7}\right) \frac{1}{s}+\left(L_{6} L_{5}+L_{7} L_{4}+L_{8}\right) \frac{1}{s^{2}}\right] \\
+\frac{e^{-R_{1}\left(\frac{s}{v_{2}}+\mu_{2}\right)}}{R^{3} a_{4}\left(\frac{1}{v_{1}^{2}}-\frac{1}{v_{2}^{2}}\right)} \cdot\left[L_{6}^{\prime}{ }_{6}+\left(L_{6}^{\prime} L_{4}+L^{\prime}{ }_{7}\right) \frac{1}{s}+\left(L_{6}^{\prime} L_{5}+L^{\prime}{ }_{7} L_{4}+L^{\prime}{ }_{8}\right) \frac{1}{s^{2}}\right]
\end{aligned}
$$

where

$$
\begin{gathered}
L_{1}, L^{\prime}{ }_{1}=P_{0} R^{2}\left(1-\frac{1}{v_{2,1}^{2}}\right)\left(a_{4}-\frac{1}{v_{1,2}^{2}}\right), \\
L_{2}, L_{2}^{\prime}=-\left[\frac{P_{0} R}{v_{1,2}} \cdot\left(2 R \mu_{1,2}+1\right)\left(1-\frac{1}{v_{2,1}^{2}}\right)+R^{2}\left(a_{4}-\frac{1}{v_{1,2}^{2}}\right)\left(\frac{2 P_{0} \mu_{2,1}}{v_{2,1}}+a_{1} a_{4} \chi\right)\right],
\end{gathered}
$$




$$
\begin{aligned}
& L_{3}, L_{3}^{\prime}=-\left[P_{0}\left(1-\frac{1}{v_{2,1}^{2}}\right)\left\{\frac{R^{2}}{v_{1,2}^{2}}\left(\mu_{1,2}^{2} \cdot v_{1,2}^{2}+2 \eta_{1,2}^{\prime} v_{1,2}\right)+R \mu_{1,2}+1\right\}\right. \\
& +R^{2}\left(a_{4}-\frac{1}{v_{1,2}^{2}}\right)\left\{P_{0}\left(\mu_{2,1}^{2}+\frac{2 \eta_{2,1}^{\prime}}{v_{2,1}}\right)+2 \chi \frac{a_{1}}{v_{2,1}}\right\}-\frac{R}{v_{1,2}} \cdot\left(2 R \mu_{1,2}+1\right) \\
& \left.\cdot\left(\frac{2 P_{0} \mu_{2,1}}{v_{2,1}}+\chi a_{1} a_{4}\right)\right] \\
& L_{4}=-\left[2 a_{4}\left(\frac{\mu_{1}}{v_{1}}-\frac{\mu_{2}}{v_{2}}\right)+2\left(\frac{1}{v_{1}}-\frac{1}{v_{2}}\right)\left(1+\frac{1}{v_{1} v_{2}}\right)\right]\left[a_{4}\left(\frac{1}{v_{1}^{2}}-\frac{1}{v_{2}^{2}}\right)\right], \\
& L_{5}=-\left[a_{4}\left(\mu_{1}^{2}-\mu_{2}^{2}+\frac{2 \eta_{1}^{\prime}}{v_{1}}-\frac{2 \eta_{2}^{\prime}}{v_{2}}\right)+2\left(\frac{1}{v_{1}^{2}}-\frac{1}{v_{2}^{2}}\right)+2\left(\mu_{1}-\mu_{2}\right)\left(1+\frac{1}{v_{1} v_{2}}\right)\right. \\
& \left.+2\left(\frac{1}{v_{1}}-\frac{1}{v_{2}}\right)\left(\frac{\mu_{1}}{v_{2}}+\frac{\mu_{2}}{v_{1}}\right)\right]\left[a_{4}\left(\frac{1}{v_{1}^{2}}-\frac{1}{v_{2}^{2}}\right)\right]+L_{4}^{2}, \\
& L_{6}, L_{6}^{\prime}=a_{4} P_{0} R^{2}\left(1-\frac{1}{v_{2,1}^{2}}\right) \\
& L_{7}, L^{\prime}{ }_{7}=\frac{2 P_{0} R}{v_{1,2}}\left(1-v_{2,1}^{2}\right)-a_{4} R^{2}\left(2 P_{0} \cdot \frac{\mu_{2,1}}{v_{2,1}}+\chi a_{1} a_{4}\right) \\
& L_{8}, L_{8}^{\prime}=P_{0}\left(1-\frac{1}{v_{2,1}^{2}}\right)\left(2 R \mu_{1,2}+2\right)-2 R\left(2 P_{0} \cdot \frac{\mu_{2,1}}{v_{2,1}}+\chi a_{1} a_{4}\right) / v_{1,2} \\
& -a_{4} R^{2}\left\{P_{0}\left(\mu_{2,1}^{2}+\frac{2 \eta_{2,1}^{\prime}}{v_{2,1}}\right)+2 \frac{\chi a_{1}}{v_{2,1}}\right\}
\end{aligned}
$$


Similarly, the expressions for $\bar{U}$ and $\bar{\odot}$ can be obtained in the form

$$
\begin{gathered}
\bar{U} \sim \frac{e^{-R_{1}\left(\mu_{1}+\frac{s}{v_{1}}\right)}}{R^{2} a_{4}\left(\frac{1}{v_{1}^{2}}-\frac{1}{v_{2}^{2}}\right)} \cdot\left[\frac{L_{13}}{s}+\left(L_{14}+L_{4} L_{13}\right) \cdot \frac{1}{s^{2}}\right] \\
-\frac{e^{-R_{1}\left(\mu_{2}+\frac{s}{v_{2}}\right)}}{R^{2} a_{4}\left(\frac{1}{v_{1}^{2}}-\frac{1}{v_{2}^{2}}\right)} \cdot\left[\frac{L_{13}^{\prime}}{s}+\left(L^{\prime}{ }_{14}+L_{4} L^{\prime}{ }_{13}\right) \frac{1}{s^{2}}\right],
\end{gathered}
$$

and

$$
\begin{gathered}
\bar{~} \sim \frac{e^{-R_{1}\left(\mu_{1}+\frac{s_{1}}{v_{1}}\right)}}{a_{1} a_{4} R\left(\frac{1}{v_{1}^{2}}-\frac{1}{v_{2}^{2}}\right)} \cdot\left[L_{15}+\left(L_{4} L_{15}+L_{16}\right) \cdot \frac{1}{s}+\left(L_{5} L_{15}+L_{4} L_{16}+L_{17}\right) \frac{1}{s^{2}}\right] \\
-\frac{e^{-R_{1}\left(\mu_{2}+\frac{1}{v_{2}}\right)}}{a_{1} a_{4} R\left(\frac{1}{v_{1}^{2}}-\frac{1}{v_{2}^{2}}\right)} \cdot\left[L^{\prime}{ }_{15}+\left(L_{4} L^{\prime}{ }_{15}+L^{\prime}{ }_{16}\right) \frac{1}{s}+\left(L_{5} L^{\prime}{ }_{15}+L_{4} L^{\prime}{ }_{16}+L^{\prime}{ }_{17}\right) \frac{1}{s^{2}},\right.
\end{gathered}
$$

where

$$
\begin{aligned}
L_{13}, L_{13}^{\prime}= & P_{0}\left(1-\frac{1}{v_{2,1}^{2}}\right) \cdot \frac{R}{v_{1,2}} \\
L_{14}, L_{14}^{\prime}= & P_{0}\left(1-1 / v_{2,1}^{2}\right)\left(R \mu_{1,2}+1\right)-R\left(2 P_{0} \mu_{2,1} / v_{2,1}+\chi a_{1} a_{4}\right) / v_{1,2} \\
L_{15}, L_{15}^{\prime}= & P_{0}\left(1-1 / v_{2,1}^{2}\right)\left(1-1 / v_{1,2}^{2}\right) \\
L_{16}, L_{16}^{\prime}= & -2 P_{0}\left(1-1 / v_{2,1}^{2}\right) \mu_{1,2} / v_{1,2}-\left(1-1 / v_{1,2}^{2}\right)\left(2 P_{0} \mu_{2,1} / v_{2,1}+\chi a_{1} a_{4}\right) \\
L_{17}, L_{17}^{\prime}= & -P_{0}\left(1-1 / v_{2,1}^{2}\right)\left(\mu_{1,2}^{2}+2 \eta_{1,2}^{\prime} / v_{1,2}\right)+2 \mu_{1,2}\left(2 P_{0} \mu_{2,1} / v_{2,1}+\chi a_{1} a_{4}\right) / v_{1,2} \\
& -\left(1-1 / v_{1,2}^{2}\right)\left\{P_{0}\left(\mu_{2,1}^{2}+2 \eta_{2,1}^{\prime} / v_{2,1}\right)+2 \chi a_{1} / v_{2,1}\right\}
\end{aligned}
$$


Application of the inverse Laplace transform [13] to (3.24)-(3.25) and (3.34)-(3.35) yields

$$
\begin{aligned}
\sigma_{\theta}=\sigma_{\phi} \sim & -\frac{e^{-\mu_{1} R_{1}}}{R^{3} a_{4}\left(1 / v_{1}^{2}-1 / v_{2}^{2}\right)} \cdot\left[L_{1} \delta\left(\eta-\frac{R_{1}}{v_{1}}\right)+\left(L_{1} L_{4}+L_{2}\right) H\left(\eta-\frac{R_{1}}{v_{1}}\right)\right. \\
& \left.+\left(L_{3}+L_{1} L_{5}+L_{2} L_{4}\right)\left(\eta-\frac{R_{1}}{v_{1}}\right) H\left(\eta-\frac{R_{1}}{v_{1}}\right)\right] \\
& +\frac{e^{-\mu_{2} R_{1}}}{R^{3} a_{4}\left(1 / v_{1}^{2}-1 / v_{2}^{2}\right)}\left[L^{\prime} \delta\left(\eta-\frac{R_{1}}{v_{2}}\right)+\left(L^{\prime}{ }_{1} L_{4}+L^{\prime}{ }_{2}\right) H\left(\eta-\frac{R_{1}}{v_{2}}\right)\right. \\
& \left.+\left(L^{\prime}{ }_{3}+L^{\prime}{ }_{1} L_{5}+L^{\prime}{ }_{2} L_{4}\right)\left(\eta-\frac{R_{1}}{v_{2}}\right) H\left(\eta-\frac{R_{1}}{v_{2}}\right)\right],
\end{aligned}
$$

and

$$
\begin{aligned}
& \sigma_{R} \sim-\frac{e^{-\mu_{1} R_{1}}}{R^{3} a_{4}\left(1 / v_{1}^{2}-1 / v_{2}^{2}\right)} \cdot\left[L_{6} \delta\left(\eta-\frac{R_{1}}{v_{1}}\right)+\left(L_{6} L_{4}+L_{7}\right) H\left(\eta-\frac{R_{1}}{v_{1}}\right)\right. \\
& \left.+\left(L_{6} L_{5}+L_{7} L_{4}+L_{8}\right)\left(\eta-\frac{R_{1}}{v_{1}}\right) H\left(\eta-\frac{R_{1}}{v_{1}}\right)\right] \\
& +\frac{e^{-\mu_{2} R_{1}}}{R^{3} a_{4}\left(1 / v_{1}^{2}-1 / v_{2}^{2}\right)} \cdot\left[L_{6}^{\prime} \delta\left(\eta-\frac{R_{1}}{v_{2}}\right)+\left(L^{\prime} L_{6}+L_{7}^{\prime}\right) H\left(\eta-\frac{R_{1}}{v_{2}}\right)\right. \\
& \left.+\left(L_{6}^{\prime} L_{5}+L_{7}^{\prime} L_{4}+L_{8}^{\prime}\right)\left(\eta-\frac{R_{1}}{v_{2}}\right) H\left(\eta-\frac{R_{1}}{v_{2}}\right)\right] \text {. } \\
& U \sim \frac{e^{-\mu_{1} R_{1}}}{R^{2} a_{4}\left(\frac{1}{v_{1}^{2}}-\frac{1}{v_{2}^{2}}\right)} \cdot\left[L_{13} H\left(\eta-\frac{R_{1}}{v_{1}}\right)+\left(L_{14}+L_{4} L_{13}\right)\left(\eta-\frac{R_{1}}{v_{1}}\right) H\left(\eta-\frac{R_{1}}{v_{1}}\right)\right] \\
& -\frac{e^{-\mu_{2} R_{1}}}{R^{2} a_{4}\left(\frac{1}{v_{1}^{2}}-\frac{1}{v_{2}^{2}}\right)} \cdot\left[L^{\prime}{ }_{13} H\left(\eta-\frac{R_{1}}{v_{2}}\right)+\left(L^{\prime}{ }_{14}+L_{4} L^{\prime}{ }_{13}\right)\left(\eta-\frac{R_{1}}{v_{2}}\right) H\left(\eta-\frac{R_{1}}{v_{2}}\right)\right] \text {, }
\end{aligned}
$$

and 


$$
\begin{aligned}
& \odot \sim \frac{e^{-\mu_{1} R_{1}}}{R a_{1} a_{4}\left(\frac{1}{v_{1}^{2}}-\frac{1}{v_{2}^{2}}\right)} \cdot\left[L_{15} \delta\left(\eta-\frac{R_{1}}{v_{1}}\right)+\left(L_{16}+L_{4} L_{15}\right) H\left(\eta-\frac{R_{1}}{v_{1}}\right)\right. \\
& \left.+\left(L_{17}+L_{4} L_{16}+L_{5} L_{15}\right)\left(\eta-\frac{R_{1}}{v_{1}}\right) H\left(\eta-\frac{R_{1}}{v_{1}}\right)\right] \\
& \quad-\frac{e^{-\mu_{2} R_{1}}}{R a_{1} a_{4}\left(\frac{1}{v_{1}^{2}}-\frac{1}{v_{2}^{2}}\right)} \cdot\left[L^{\prime}{ }_{15} \delta\left(\eta-\frac{R_{1}}{v_{2}}\right)+\left(L^{\prime}{ }_{16}+L_{4} L^{\prime}{ }_{15}\right) H\left(\eta-\frac{R_{1}}{v_{2}}\right)\right. \\
& \left.+\left(L^{\prime}{ }_{17}+L_{4} L^{\prime}{ }_{16}+L_{5} L^{\prime}{ }_{15}\right)\left(\eta-\frac{R_{1}}{v_{2}}\right) H\left(\eta-\frac{R_{1}}{v_{2}}\right)\right] .
\end{aligned}
$$

CASE II. Proceeding as in Case I, for small values of time, we obtain

$$
\begin{aligned}
\sigma_{\theta}=\sigma_{\phi} \sim & -\frac{e^{-\mu_{1} R_{1}}}{R^{3} a_{4}\left(\frac{1}{v_{1}^{2}}-\frac{1}{v_{2}^{2}}\right)} \cdot\left[L_{9} H\left(\eta-\frac{R_{1}}{v_{1}}\right)+\left(L_{10}+L_{4} L_{9}\right)\left(\eta-\frac{R_{1}}{v_{1}}\right) \cdot H\left(\eta-\frac{R_{1}}{v_{1}}\right)\right] \\
& +\frac{e^{-\mu_{2} R_{1}}}{R^{3} a_{4}\left(\frac{1}{v_{1}^{2}}-\frac{1}{v_{2}^{2}}\right)} \cdot\left[L^{\prime}{ }_{9} H\left(\eta-\frac{R_{1}}{v_{2}}\right)+\left(L^{\prime}{ }_{10}+L_{4} L_{9}^{\prime}\right)\left(\eta-\frac{R_{1}}{v_{2}}\right) H\left(\eta-\frac{R_{1}}{v_{2}}\right)\right],
\end{aligned}
$$

and

$$
\begin{aligned}
\sigma_{R} \sim & -\frac{e^{-\mu_{1} R_{1}}}{R^{3} a_{4}\left(\frac{1}{v_{1}^{2}}-\frac{1}{v_{2}^{2}}\right)} \cdot\left[L_{11} H\left(\eta-\frac{R_{1}}{v_{1}}\right)+\left(L_{12}+L_{4} L_{11}\right)\left(\eta-\frac{R_{1}}{v_{1}}\right) \cdot H\left(\eta-\frac{R_{1}}{v_{1}}\right)\right] \\
& +\frac{e^{-\mu_{2} R_{1}}}{R^{3} a_{4}\left(\frac{1}{v_{1}^{2}}-\frac{1}{v_{2}^{2}}\right)} \cdot\left[L^{\prime}{ }_{11} H\left(\eta-\frac{R_{1}}{v_{2}}\right)+\left(L^{\prime}{ }_{12}+L_{4} L^{\prime}{ }_{11}\right)\left(\eta-\frac{R_{1}}{v_{2}}\right) \cdot H\left(\eta-\frac{R_{1}}{v_{2}}\right)\right],
\end{aligned}
$$

where 


$$
\begin{aligned}
L_{9}, L_{9}^{\prime}= & R^{2}\left\{P_{0}^{\prime}\left(1-1 / v_{2,1}^{2}\right)-\chi a_{1} a_{4}\right\} \cdot\left(a_{4}-1 / v_{1,2}^{2}\right), \\
L_{10}, L^{\prime}{ }_{10}= & -\left\{P_{0}^{\prime}\left(1-1 / v_{2,1}^{2}\right)-\chi a_{1} a_{4}\right\} \cdot\left\{2 R^{2} \mu_{1,2} / v_{1,2}+R / v_{1,2}\right\} \\
& -R^{2}\left(a_{4}-1 / v_{1,2}^{2}\right)\left(2 P^{\prime} \mu_{2,1} / v_{2,1}+2 \chi a_{1} / v_{2,1}\right), \\
L_{11}, L_{11}^{\prime}= & a_{4} R^{2}\left\{P_{0}^{\prime}\left(1-1 / v_{2,1}^{2}\right)-\chi a_{1} a_{4}\right\} \\
L_{12}, L_{12}^{\prime}= & 2 R\left\{P_{0}^{\prime}\left(1-1 / v_{2,1}^{2}\right)-\chi a_{1} a_{4}\right\} / v_{1,2}-a_{4} R^{2}\left\{\left(2 P^{\prime}{ }_{0} \mu_{2,1}+2 \chi a_{1}\right) / v_{2,1}\right\}
\end{aligned}
$$

We can also find

$$
\begin{gathered}
\bar{U} \sim \frac{e^{-R_{1}\left(\mu_{1}+s N_{1}\right)}}{R^{2} a_{4}\left(\frac{1}{v_{1}^{2}}-\frac{1}{v_{2}^{2}}\right)} \cdot \frac{L_{18}}{s^{2}}-\frac{e^{-R_{1}\left(\mu_{2}+s N_{2}\right)}}{R^{2} a_{4}\left(\frac{1}{v_{1}^{2}}-\frac{1}{v_{2}^{2}}\right)} \cdot \frac{L_{18}^{\prime}}{s^{2}}, \\
\bar{\odot} \sim \frac{e^{-R_{1}\left(\mu_{1}+s N_{1}\right)}}{R a_{1} a_{4}\left(\frac{1}{v_{1}^{2}}-\frac{1}{v_{2}^{2}}\right)} \cdot\left[\frac{L_{19}}{s}+\left(L_{20}+L_{4} L_{19}\right) \cdot \frac{1}{s^{2}}\right] \\
-\frac{e^{-R_{1}\left(\mu_{2}+s N_{2}\right)}}{R a_{1} a_{4}\left(\frac{1}{v_{1}^{2}}-\frac{1}{v_{2}^{2}}\right)} \cdot\left[\frac{L_{19}^{\prime}}{s}+\left(L_{20}^{\prime}+L_{4} L_{19}^{\prime}\right) \cdot \frac{1}{s^{2}}\right]
\end{gathered}
$$

where

$$
\begin{gathered}
L_{18}, L_{18}^{\prime}=\frac{R}{v_{1,2}} \cdot\left[P_{0}^{\prime}\left(1-1 / v_{2,1}^{2}\right)-\chi a_{1} a_{4}\right], \\
L_{19}, L_{19}^{\prime}=\left(1-1 / v_{1,2}^{2}\right)\left[P_{0}^{\prime}\left(1-1 / v_{2,1}^{2}\right)-\chi a_{1} a_{4}\right], \\
L_{20}, L_{20}^{\prime}=-\frac{2 \mu_{1,2}}{v_{1,2}} \cdot\left[P_{0}^{\prime}\left(1-1 / v_{2,1}^{2}\right)-\chi a_{1} a_{4}\right]-\left(1-1 / v_{1,2}^{2}\right)\left[2 P^{\prime}{ }_{0} \frac{\mu_{2,1}}{v_{2,1}}+\frac{2 \chi a_{1}}{v_{2,1}}\right] .
\end{gathered}
$$


Finally, we obtain the approximate solutions in the form

$$
\begin{gathered}
U \sim \frac{e^{-\mu_{1} R_{1}}}{R^{2} a_{4}\left(\frac{1}{v_{1}^{2}}-\frac{1}{v_{2}^{2}}\right)} \cdot L_{18}\left(\eta-\frac{R_{1}}{v_{1}}\right) \cdot H\left(\eta-\frac{R_{1}}{v_{1}}\right) \\
-\frac{e^{-\mu_{2} R_{1}}}{R^{2} a_{4}\left(\frac{1}{v_{1}^{2}}-\frac{1}{v_{2}^{2}}\right)} \cdot L^{\prime}{ }_{18}\left(\eta-\frac{R_{1}}{v_{2}}\right) \cdot H\left(\eta-\frac{R_{1}}{v_{2}}\right), \\
\odot \approx \frac{e^{-\mu_{1} R_{1}}}{R a_{1} a_{4}\left(\frac{1}{v_{1}^{2}}-\frac{1}{v_{2}^{2}}\right)} \cdot\left[L_{19} H\left(\eta-\frac{R_{1}}{v_{1}}\right)+\left(L_{20}+L_{4} L_{19}\right)\left(\eta-\frac{R_{1}}{v_{1}}\right) \cdot H\left(\eta-\frac{R_{1}}{v_{1}}\right)\right] \\
-\frac{e^{-\mu_{2} R_{1}}}{R a_{1} a_{4}\left(\frac{1}{v_{1}^{2}}-\frac{1}{v_{2}^{2}}\right)} \cdot\left[L^{\prime}{ }_{19} H\left(\eta-\frac{R_{1}}{v_{2}}\right)+\left(L^{\prime}{ }_{20}+L_{4} L^{\prime}{ }_{19}\right)\left(\eta-\frac{R_{1}}{v_{2}}\right) \cdot H\left(\eta-\frac{R_{1}}{v_{2}}\right)\right]
\end{gathered}
$$

\section{DISCUSSION AND CONCLUSIONS}

It follows from the above short time approximation of solutions that the disturbances consist of two types of waves propagating with finite velocities $v_{1}$ and $v_{2}\left(>v_{1}\right)$. In the limit $\tilde{\beta} \rightarrow 0, v_{1} \rightarrow 1$ and $v_{2} \rightarrow \infty$. This limit corresponds to the classical coupled theory which predicts an infinite speed of thermal wave. Obviously, $v_{2}$ is the speed of thermal wave and hence $v_{1}$ is the speed of elastic wave. Since $v_{2}>v_{1}$, the elastic wave follows the thermal wave. It may be pointed out that the forms of $\psi_{1}(p)$ and $\psi_{2}(p)$ suggest that there is no diffusive nature of the waves as found in Nariboli [11]. This is one of the significant features of the present study.

The present analysis also reveals that stress, displacement and temperature are found to experience discontinuities at the wavefronts, and they are given below. For Case I, the discontinuities are

$$
\left[\sigma_{\theta}^{+}-\sigma_{\theta}^{-}\right]_{R_{1}-\eta v_{1,2}}=\mp \frac{e^{-\mu_{1,2} R_{1}}}{R^{3} a_{4}\left(\frac{1}{v_{1}^{2}}-\frac{1}{v_{2}^{2}}\right)} \cdot\left[L_{4}\left(L_{1}, L_{1}^{\prime}\right)+\left(L_{2}, L_{2}^{\prime}\right)\right],
$$




$$
\begin{aligned}
& {\left[\sigma_{R}^{+}-\sigma_{R}^{-}\right]_{R_{1}=\eta v_{1,2}}=\mp \frac{e^{-\mu_{1,2} R_{1}}}{R^{3} a_{4}\left(\frac{1}{v_{1}^{2}}-\frac{1}{v_{2}^{2}}\right)} \cdot\left[L_{4}\left(L_{6}, L_{6}^{\prime}\right)+\left(L_{7}, L_{7}^{\prime}\right)\right],} \\
& {\left[U^{+}-U^{-}\right]_{R_{1}-\eta v_{1,2}}= \pm \frac{e^{-\mu_{1,2} R_{1}}}{R^{2} a_{4}\left(\frac{1}{v_{1}^{2}}-\frac{1}{v_{2}^{2}}\right)} \cdot\left[L_{13}, L_{13}^{\prime}\right],}
\end{aligned}
$$

and

$$
\left[\odot^{+}-\odot^{-}\right]_{R_{1}-\eta v_{1,2}}= \pm \frac{e^{-\mu_{1,2} R_{1}}}{R^{2} a_{4}\left(\frac{1}{v_{1}^{2}}-\frac{1}{v_{2}^{2}}\right)} \cdot\left[\left(L_{16}, L_{16}^{\prime}\right)+L_{4} \cdot\left(L_{15}, L_{15}^{\prime}\right)\right] .
$$

For Case II, the displacement is continuous at the wavefront. However, the stress and temperature are discontinuous at the wavefronts, and are given by

$$
\begin{aligned}
& {\left[\sigma_{\theta}^{+}-\sigma_{\theta}^{-}\right]_{R_{1}-\eta v_{1,2}}=\mp \frac{e^{-\mu_{1,2} R_{1}}}{R^{3} a_{4}\left(\frac{1}{v_{1}^{2}}-\frac{1}{v_{2}^{2}}\right)}\left[L_{9}, L_{9}^{\prime}\right],} \\
& {\left[\sigma_{R}^{+}-\sigma_{R}^{-}\right]_{R_{1}-\eta v_{1,2}}=\mp \frac{e^{-\mu_{1,2} R_{1}}}{R^{3} a_{4}\left(\frac{1}{v_{1}^{2}}-\frac{1}{v_{2}^{2}}\right)} \cdot\left[L_{11}, L_{11}^{\prime}\right],}
\end{aligned}
$$

and

$$
\left[\odot^{+}-\odot^{-}\right]_{R_{1}-\eta v_{1}, 2}= \pm \frac{e^{-R_{1} \mu_{1,2}}}{R a_{1} a_{4}\left(\frac{1}{v_{1}^{2}}-\frac{1}{v_{2}^{2}}\right)} \cdot\left[L_{19}, L_{19}^{\prime}\right]
$$

ACKNOWLEDGMENT. This research was partially supported by the University of Central Florida.

\section{REFERENCES}

[1] Lord, H.W. and Shulman, Y., A Generalized Dynamical Theory of Thermoelasticity, J. Mech. Phys. Solids, 15(1967), 299-309.

[2] Boley, B.A. and Weiner, J.H., Theory of Thermal Stress, John Wiley, New York, (1960).

[3] Norwood, F.R. and Warren. W.E., Wave Propagation in the Generalized Dynamical Theory of Thermoelasticity, Quart.J. Mech. Appl. Math., 22(1969), 283-290. 
[4] McCarthy, M.F., Wave Propagation in Generalized Thermoelasticity, Int. J. Eng. Sci., 10(1972), 593-602.

[5] Puri, P., Plane Waves in Generalized Thermoelasticity, Int.J. Eng. Sci., 11(1973), 735-744.

[6] Agarwal, V.K., On Plane Waves in Generalized Thermoelasticity, Acta. Mech., 31(1979), 185-198.

[7] Chandrasekharaiah, D.S., On Generalized Thermoelastic Wave Propagation, Proc. Indian Acad. Sci., (Math. Sci.), 89(1980), 43-52.

[8] Chandrasekharaiah, D.S., Srinath, K.S., and Debnath, L., Magneto-Thermo-Elastic Disturbances With Thermal Relaxation in a Solid Due to Heat Sources, Comput. Math. Applic.. 15(1988), 483-490.

[9] Roy-Choudhuri, S.K. and Debnath, L., Magnetoelastic Plane Waves in Infinite Rotating Media, J. Appl. Mech. Trans. ASME, 106(1984), 283-287.

[10] Roy-Chaudhuri, S.K. and Debnath, L., Magneto-Thermo-Elastic Plane Waves in Rotating Media, Int. J. Engg. Sci., 21(1983), 155-163.

[11] Nariboli, G.A., Spherically Symmetric Thermal Shock in a Medium With Thermal And Elastic Deformations Coupled, Quart. J. Appl. Mech., 14(1961), 75-84.

[12] Matsumoto, H. and Nakahara, I., Hollow Sphere Subjected to Dynamic Pressure on its Inner or Outer Surface, _Bull, Tokyo Ins. Tech., 109(1971), 27-55.

[13] Myint-U, T., and Debnath, L., Partial Differential Equations for Scientists and Engineers (Third Edition) North Holland, New York (1987).

[14] Doetsch, G. Introduction to the Theory And Application of the Laplace Transformation, (Translated by Walter Nader) Springer Verlag, New York (1970). 


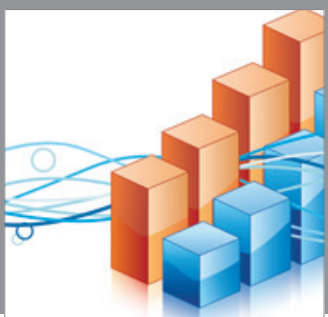

Advances in

Operations Research

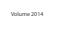

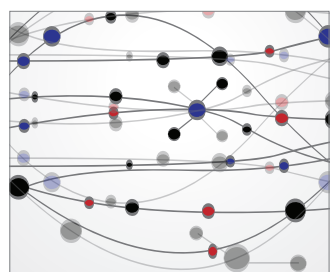

\section{The Scientific} World Journal
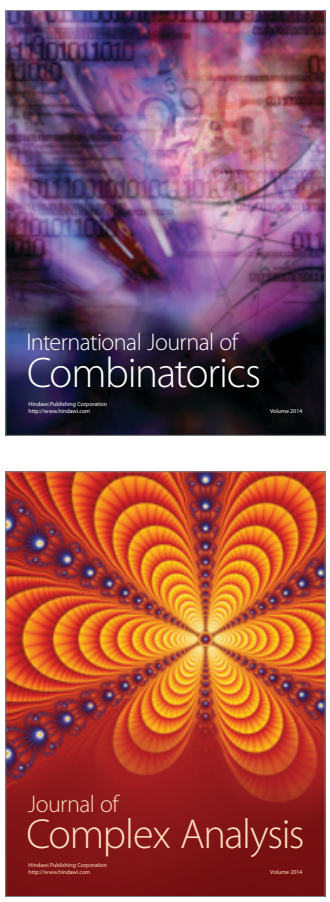

International Journal of

Mathematics and

Mathematical

Sciences
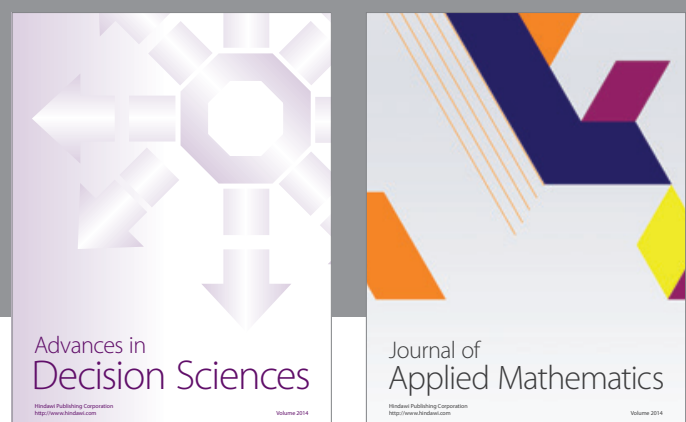

Journal of

Applied Mathematics
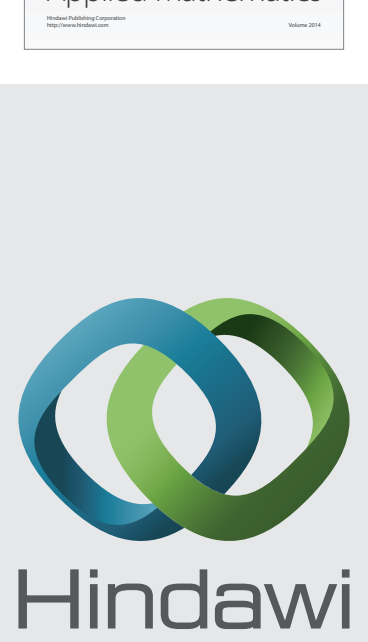

Submit your manuscripts at http://www.hindawi.com
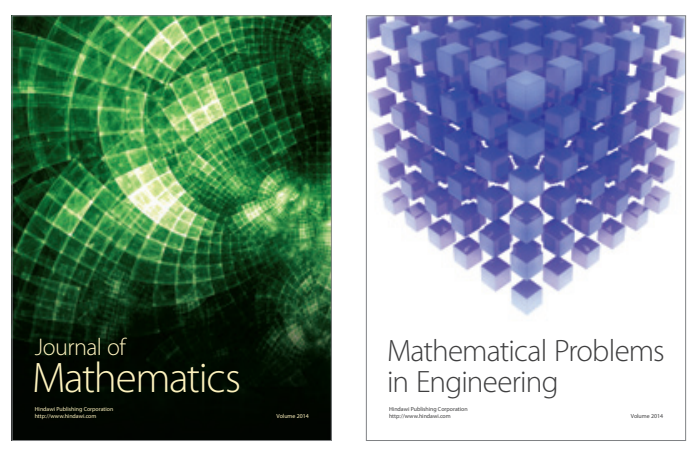

Mathematical Problems in Engineering
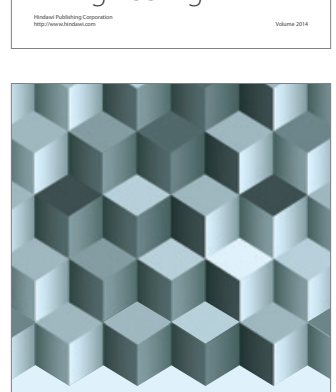

Journal of

Function Spaces
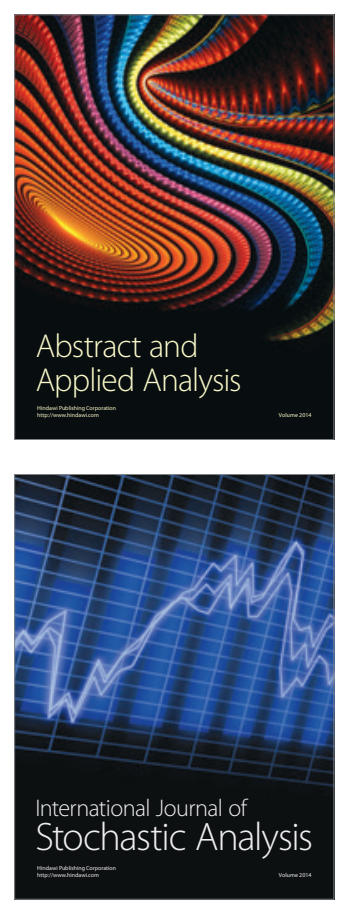

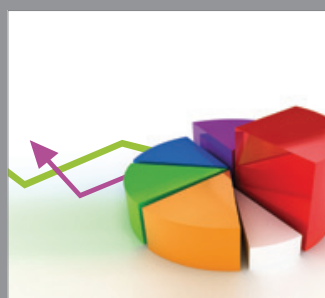

ournal of

Probability and Statistics

Promensencen
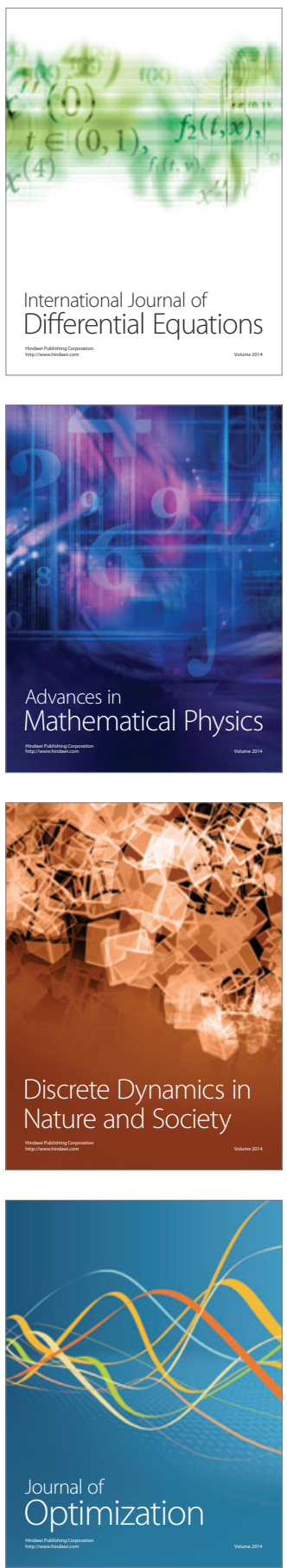